\title{
LA TRANSFORMADA DE LAPLACE EN LA SOLUCIÓN DE ECUACIONES DIFERENCIALES CON ALGORITMO DE CÁLCULO EN MATHCAD
}

\section{The laplace transformation in the solution of differential equations with calculation algorithms in mathcad}

JUAN ANTONIO MARTIN ALFONSO [1], CARLOS MANUEL MATA RODRÍGUEZ [2]

Recibido:03 de junio de 2019. A ceptado:20 de junio de 2019

DOI: http:/ / dx.doi.org/ 10.21017/ rimci.2019.v6.n12.a64

\begin{abstract}
Resumen
En la historia de las matemáticas, ocurre con frecuencia que determinados temas (en especial el cálculo numérico) tenían perfectamente definida su solución teórica, pero el cúmulo de operaciones para hallar un resultado concluyente, en múltiples ocasiones no permitía llegar a los resultados finales.

La solución de las ecuaciones diferenciales, representa un ejemplo demostrativo.

A finales del siglo XIX la electricidad era tema fundamental en la sociedad y cada vez aparecían nuevas situaciones que complejizaban la solución de problemas técnicos, en especial con la teoría de los circuitos eléctricos que para llegar a soluciones finales era necesario resolver ecuaciones diferenciales por los métodos clásicos haciendo uso intenso de las técnicas de integración y deriva ción lo cual constituía en múltiples ocasiones un notable obstáculo desde el punto de vista ingenieril, y fue a finales del siglo cuando un ingeniero electricista ingles estableció un conjunto de reglas prácticas para llegar a dichas soluciones sin la necesidad de utilizar los fundamentos del cálculo. Si bien estas reglas propiciaban las soluciones, era necesario efectuar complejas operaciones algebraicas en ocasiones largas y tediosas, por lo que comparativamente no quedaba claro cual proceder sería el mejor.

En la actualidad con el apoyo software matemático, en especial Mathcad, podemos llegar a dichas soluciones de manera rápida y con un alto grado de precisión. Y es sobre este aspecto que trata el trabajo que presentamos en el cual se describe un procedimiento algorítmico que permite hallar la solución de ecuaciones diferenciales con condiciones iníciales, aplicando la transformada de Laplace.
\end{abstract}

Palabras clave: Ecuaciones diferenciales, Transformada de Laplace, movimiento vibratorio, circuitos eléctricos.

\begin{abstract}
In the history of Mathematics, frequently happens that certain to pics (especially the numeric calculation) has perfectly defined its theoretical solution, but the heap of operations to find a conclusive result, in multiple occasions, don'tallow to arrive to the final results.

The solution of differential equations represents a demonstrative example. At the end of the XIX century, the electricity was a fundamental theme in the society and every time new situations appeared that complicates the solution of technical problems, especially with the theory of the electric circuits, where was necessary solving differential equations with classic methods, making intense use of the techniques of integration and derivation, which constituted, in multiple occasions, a remarkable obstacle from the engendering point of view, and it was at the end of the century when an English electrical engineer established a group of practical rules to arrive to this solutions without necessity of using the basics of calculus.
\end{abstract}

[1] Licenciado en Matemática y Doctor en Ciencias Físicas, se desempeña como jefe del Departamento de Matemática de la U niversidad de Ciego de Á vila "Máximo Gómez Báez". Con varias publicaciones en el estudio de la cavidades ópticas de Láseres Semiconductores, en problemas relacionados con la enseñanza de la M atemática y en Informática A plicada.

[2] Profesor Licenciado en Matemáticas. Consultor para la Formación de Personal en Informática. Miembro de la A NIR (A sociación N acional de Inventores y Racionalizadore). A ctualmente Departamento de Matemáticas, Universidad de Ciego de Á vila. Cuba. Correo: camaro@ unica.cu 
Although these rules propitiated the solutions, was necessary to do complex algebraic operations, in occasions, long and tedious, so, comparatively, it was not clear which procedure would be the best.

At the present time, with the support of mathematical software, especially Mathcad, we can arrive to this solution in a quick way and with a high grade of precision.

In the present text is described an algo rithmic procedure to find the solution of differential equations with initial conditions, applying the transformed of Laplace.

Key words: differential equations, Laplace transforms, vibrations of a mass on a spring, electric circuits.

\section{INTRODUCCIÓN}

E interesantes que disponemos para analizar y pre decir el comportamiento de un sistema o fenómeno de diferente naturaleza es la construcción y posterior simulación deun modelo matemático. Los avances ocurridos en las diferentes ramas del saber humano junto al desarrollo alcanzado en los ordenadores y el software matemáticos son razones que justifican la edad de oro que hoy en día vivela modelización matemática.

Los modelos se construyen para conocer o predecir propiedades del objeto real. Según el profesor Sixto Ríos [1]: “un modelo es un objeto, concepto o conjunto de relaciones, quese utiliza para representar y estudiar de forma simpley comprensible una porción dela realidad empírica».

Para quelos modelos puedan decirnos al go sobre el objeto que representan, es necesario que se construyan estableciendo una relación con la realidad que debe ser simétrica, es decir, la relación de correspondencia entre el objeto real y el modelo debe ser al menos parcialmente reversibley debe permitir la traducción de al gunas propiedades del modelo a la realidad.

Existe una gran variedad de model os matemáticos que se utilizan para el estudio de problemas en diferentes ciencias; sus objetivos principales son describir, explicar y predecir fenómenos y procesos en dichas áreas. Una gran partedetales modelos matemáticos se expresan mediante ecuaciones diferenciales.

En particular las ecuaciones diferenciales ordinarias de segundo orden con coeficientes constantes nos permiten modelar fenómenos vibratorios y circuitos eléctricos en series.

Los asistentes matemáticos pueden tener variado uso en la enseñanza de la matemática como se- ñala Luis Zaldívar Henríquez y colaboradores [2], si se tiene en cuenta que en la solución de problemas se transitan por diferentes etapas que según Santiago Vicente y Josetxu Orrantia [3] consisten en comprensión del texto, comprensión de la situación, matematización, cálculo e interpretación del resultado. Siendo la etapa de matematización, donde seestableceel modelo matemático quedescribe la problemática planteada, crucial en dicho proceso al igual quela deinterpretación de los resultados; pero la etapa del cálculo puede ser en muchas ocasiones muy extensa y rutinaria provocando que se puedan resolver en clase un número reducido de problemas pues la mayor parte del tiempo sedediquea los cálcul os como señalan $\mathrm{Pe}$ dro Campillo y Antonio Devesa [4]. Esto haceque no se puedan desarrollar, como se desea la habilidad para solucionar problemas en los al umnos. Es aquí donde puede entrar el uso del asistente matemático como una herramienta de cálcul o que permita elevar la productividad del estudiante en la clase y se pueda dedicar la mayor parte de esta a las etapas de matematización e interpretación de los resultados y deesta forma poner a estudiantes en posición de éxito en la solución de problemas.

Esta situación es típica en el caso de los problemas que conducen a una ecuación diferencial lineal no homogénea de segundo orden con coeficientes contantes como modelo matemático, pues su proceso de solución por la vía directa requiere en primer lugar de la solución de la ecuación dife-rencial homogénea asociada, lo que requiere de la determinación de las raíces de una ecuación polinómica; una vez transcurrido esta etapa hay que determinar la solución particular dela ecuación diferencial, y cualquier método quesesiga trae con sigo un volumen significativo de cálculos al gebraicos que general mente conll levan a la solución desistemas deecuaciones lineales eincluso es necesario calcular integrales si se está usando el método de variación dela constante creado por el matemático italo-francés osé Luis Lagrange(17361813) [5].

Rev. Ingeniería, M atemáticas y Ciencias de la Información Vol. 6 / N úm. 12 / julio - diciembre de 2019; pág. 25-35 
Una vez vencida la etapa dela solución general de la ecuación diferencial se hace necesario determinar la solución particular que satisface las condicionesiníciales asociadas al problema queseestá resolviendo, proceso que requiere de cálculo de derivadas y de otro volumen significativo de cálculo al gebraico.

Una herramienta potente que permite integrar en el proceso dela solución dela ecuación diferencial el cumplimiento de las condiciones iníciales asociadas al problema es el uso de la Transformada de Laplace. La moderna aplicación de dicha transformada y toda su teoría subyacente surge en la segunda mitad del siglo XIX. Al tratar de resolver ecuaciones diferenciales relacionadas con la teoría de vibraciones, el ingeniero inglés Oliver Heaviside (1850-1925) [5][6]. Pero a pesar de esto seguimos antes la problemática de tener que enfrentar un gran volumen de cálculo para resolver la ecuación diferencial con sus respectivas condiciones iníciales que modelan el problema en cuestión, pues es necesario hacer cálculos detransformadas directas e inversa, lo que requiere de grandes volúmenes de cálculo al gebraico y gran habilidad en el uso de las propiedades y tablas de la transformada de la Laplace.

Por otra parte, el software Mathcad es un utilitario matemático que brinda amplias facilidades para el cálculo simbólico y en el mismo esta implementado todo el trabajo con la transformada directa e inversa de la Laplace. Es por ello por lo que el objetivo del presente artículo es presentar un al goritmo, implementado en M athcad, quepermite resolver analíticamente las ecuaciones diferenciales lineales con coeficientes constantes presentes en los problemas de aplicación dedichas ecuaciones y queviabiliza la etapa de cálculo dando amplias posibilidades para poder trabajar los momentos de modelación e interpretación de los resultados en el proceso de solución de esta tipología de problemas.

\section{Definición E HISTORIA DE LA TRANSFORMADA DE LAPLACE}

La teoría de las transformadas o transformaciones de Laplace, conocida tam-bién con el nombre de cál culo operacional, ha venido a constituir en Iosúltimosaños con el empleo delas computadoras, parte esencial de la matemática requerida por los ingenieros, físicos, matemáticos y otros científicos. Esto se debea que, además del interés teórico propio de la materia, los métodos de la transformada deLaplace constituyen un instrumento fácil y efectivo para la solución de muchos problemas de la ciencia y la ingeniería.

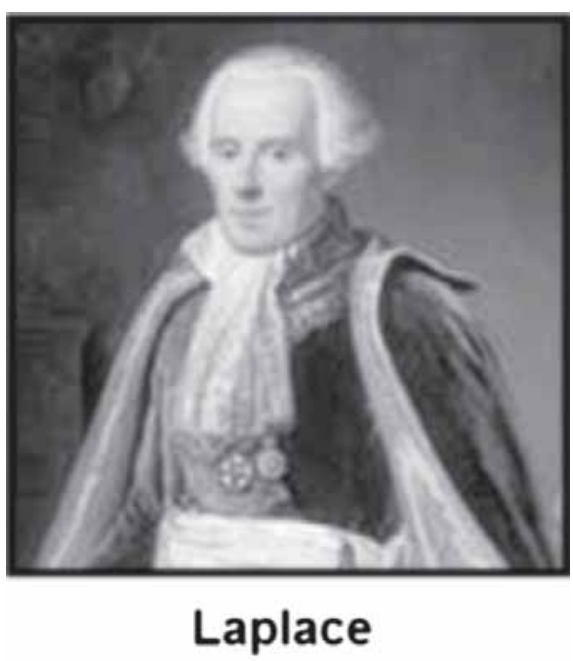

La transformada cuyo símbolo identificativo es $\mathcal{L}$ fue introducida por el matemático francés Pierre Simón Laplace (1749-1827) siguiendo las ideas de Leonardo Euler (1707-1783) en el año de 1782 [7] [8].

La transformada se fundamenta en una integral impropia de primera especie y se expresa simbólicamente como:

$$
F(s)=\mathcal{L}\{f(t)\}=\int_{0}^{\infty} e^{-s t} f(t) d t
$$

para todos los números positivos $t \geq 0$, siempre que la integral converja al menos para algún valor de s, siendo s un parámetro de variable compleja.

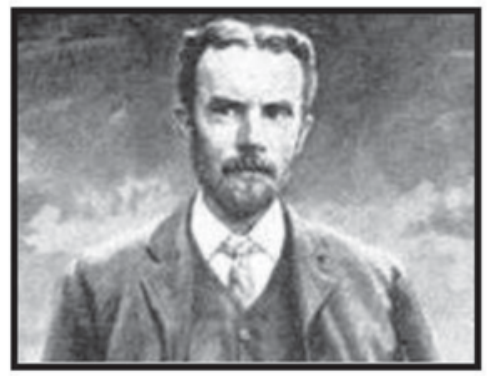

Heaviside 
Más de un siglo transcurrió sin utilización práctica de la fórmula pues fue tratada bajo una perspectiva meramente teórica hasta el año 1894, cuando el ingeniero electricista inglés Oliver Heaviside (18501925), publicó un artículo titutado "On operators in Mathematical physics" en el que introdujo ciertas reglas y métodos operatorios con muy débil justificación matemática, pero que en "general" conducían a la solución correcta de problemas sobre circuitos eléctricos.

Estos métodos, introducidos con el objeto de reducir a cuestiones puramente algebraicas la resolución de ecuaciones diferenciales y sistemas de ecuaciones diferenciales relacionados con la teoría de los circuitos eléctricos, han seguido su desarrollo, empleándose incluso por electricistas y otros técnicos, pero fueron considerados durante mucho tiempo por los matemáticos como simples medios prácticos para hallar posibles soluciones.

Tras varias décadas de intentos, se descubrió que la Transformada creada por Laplace no sólo ofrecía un fundamento teórico al método de cálculo operacional de Heaviside, sino que además permitía una alternativa mucho más sistemática en la obtención de la función solución de las ecuaciones diferenciales, siendo muy útiles para resolver ecuaciones con condiciones iníciales [9].

\section{IMPLEMENTACIÓN DEL PROCEDIMIENTO}

El uso de la transformada de Laplace en la solución de ecuaciones diferenciales está soportado en una de su ventaja más significativa de convertir la integración y derivación en multiplicación y división; para poder transformar la ecuación diferencial y sus condiciones iníciales (problema de Cauchy) hay que usar la propiedad de linealidad de la transformada:

$$
\mathcal{L}[a f(t)+b g(t)]=a \mathcal{L}[f(t)]+b \mathcal{L}[g(t)]
$$

Unido a la trasformada de la derivada, si se tiene que $L[f(t)]=F(s)$, entonces se cumple que

$$
\begin{gathered}
\mathcal{L}\left[f^{(n)}(t)\right]=s^{n} \mathcal{L}[f(t)]-\sum_{i=1}^{n} s^{n-i} f^{(i-1)}(0)= \\
s^{n} F(s)-\sum_{i=1}^{n} s^{n-i} f^{(i-1)}(0)
\end{gathered}
$$

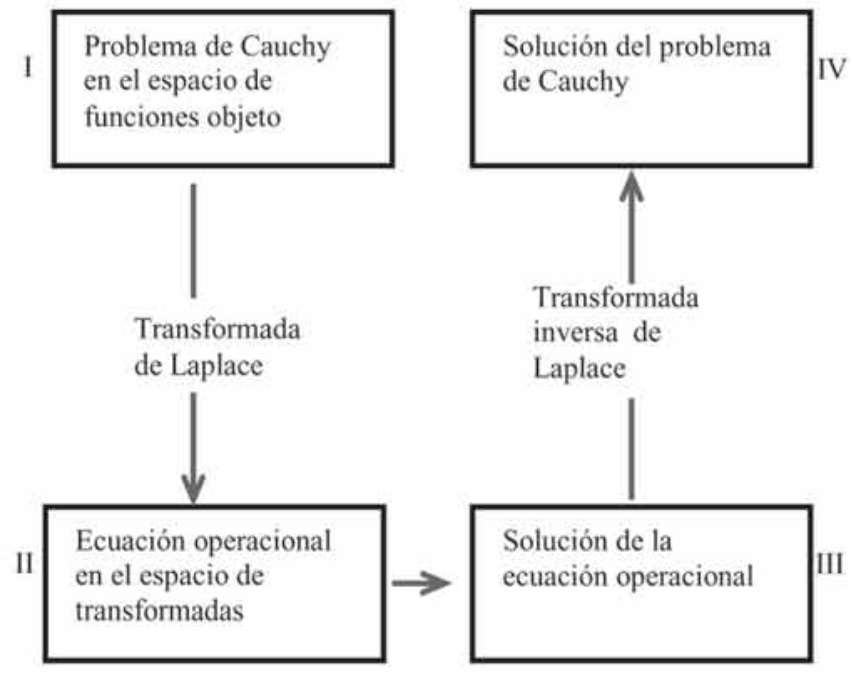

Fig.1. Esquema de cálculo para resolver un problema de Cauchy usando la transformada de Laplace

A partir de estas propiedades y usando el esquema general que se muestra en la Fig. 1, entonces es posible solucionar un problema de Cauchy:

En lo que sigue se discutirá la implementación en Mathcad [10] del procedimiento para solucionar un problema de Cauchy, en primer instancia se utiliza la propiedad (3), como se ilustra en la Fig. 2, donde k es el orden de la derivada y $\mathrm{v}$ es el vector que contiene los valores de $f(0), f^{\prime}(0), f^{\prime \prime}(0), \cdots, f^{(k-1)}(0)$, el que hay escribirlo como un vector columna.

$$
\operatorname{Tder}(\mathrm{Y}, \mathrm{s}, \mathrm{k}, \mathrm{v}):=\mid \begin{aligned}
& \mathrm{R} \leftarrow \mathrm{Y} \text { if } \mathrm{k}=0 \\
& \mathrm{R} \leftarrow \mathrm{s}^{\mathrm{k}} \cdot \mathrm{Y}-\sum_{\mathrm{i}=0}^{\mathrm{k}-1}\left(\mathrm{v}_{\mathrm{i}} \cdot \mathrm{s}^{\mathrm{k}-\mathrm{i}-1}\right) \text { otherwise }
\end{aligned}
$$

Fig. 2. Función [Tder] implementada en Mathcad para transformar la derivada de orden $\mathrm{k}$ de la función $\mathrm{f}(\mathrm{t}]$, donde los valores en $\mathrm{t}=\mathrm{O}$ de las

derivadas de orden $0,1, \ldots, k-1$ se almacenan en el vector $\mathrm{v}$.

Así por ejemplo, si se tiene que $f(0)=1$ y $f^{\prime}(0)=2$, entonces $\mathrm{v}=(1,2)^{\mathrm{T}}$, donde $\mathrm{T}$ denota la matriz transpuesta, entonces para la segunda derivada de $\mathrm{f}(\mathrm{t})$ se obtiene

$$
\operatorname{Tder}(\mathrm{Y}, \mathrm{s}, 2, \mathrm{v}) \rightarrow \mathrm{Y} \cdot \mathrm{s}^{2}-\mathrm{s}-2
$$

A partir de la función implementada para transformar una derivada y usando la propiedad de linealidad (2) se define otra función que transforma el miembro izquierdo de la ecuación diferencial, dicha función se 
muestra en la Fig. 3, en la misma aparecen dos vectores columna, uno con los coeficientes constantes (coefi) de la ecuación diferencial y otro con las condiciones iníciales. (Cini)

$$
\operatorname{MiEc}\left(\mathrm{Y}, \mathrm{s}, \text { coefi, Cini) }:=\mid \begin{array}{l}
\mathrm{n} \leftarrow \text { last }(\text { coefi }) \\
\mathrm{R} \leftarrow 0 \\
\text { for } \mathrm{i} \in 0 \ldots \mathrm{n} \\
\mathrm{R} \leftarrow \mathrm{R}+\operatorname{coefi} i_{1} \cdot \operatorname{Tder}(\mathrm{Y}, \mathrm{s}, \mathrm{i}, \text { Cini }) \\
\mathrm{R}
\end{array}\right.
$$

Fig. 3. Función implementada en Mathcad para transformar el miembro izquierdo de la ecuación diferencial en el espacio de transformadas.

Como ejemplo para la ecuación diferencial $y^{\prime \prime}-3 y^{\prime}+2 y=t e^{t}+4$ que satisface las condiciones iníciales $y(0)=1 y y^{\prime}(0)=2$ se definen los vectores columna coefi $=(2,-3,1)^{\mathrm{T}}$ de los coeficientes y el vector columna $\mathrm{Cini}=(1,2)^{\mathrm{T}}$ con las condiciones iníciales, resultando que:

$\operatorname{MiEc}(\mathrm{Y}, \mathrm{s}$, coefi, Cini) factor $\rightarrow(\mathrm{s}-1) \cdot(\mathrm{Y} \cdot \mathrm{s}-2 \cdot \mathrm{Y}-1)$

Es necesario ahora transformar el término independiente $f(t)$ (miembro derecho) de la ecuación diferencial para ello se utiliza el operador laplace $\rightarrow$ implementado en Mathcad y se define el miembro derecho de la ecuación operacional como se ilustra en la Fig. 4.

$$
\operatorname{MdEc}(\mathrm{s}):=\mathrm{f}(\mathrm{t}) \text { laplace } \rightarrow
$$

Fig. 4. Función implementada en Mathcad para transformar el miembro derecho de la ecuación diferencial en el espacio de transformadas.

Así, para el ejemplo que estamos ilustrando resulta que $f(t)=t e^{t}+4$, por lo que $\operatorname{MdEc}(s)=\frac{4 s^{2}-7 s+4}{s(s-1)^{2}}$, en la Fig.5 se muestra el resultado del cálculo realizado en Mathcad para obtener este término.

$$
\operatorname{MdEc}(s):=f(t) \text { laplace } \rightarrow \frac{4 \cdot s^{2}-7 \cdot s+4}{s \cdot(s-1)^{2}}
$$

Fig.5. Evaluación de la función para obtener la transformada del término independiente $f(t)=t e^{t}+4$ de la ecuación diferencial
Siguiendo el esquema de cálculo representado en la Fig. 1 corresponde primeramente encontrar $\mathrm{Y}$ en la ecuación:

$$
(s-1)(Y s-2 Y-1)=\frac{4 s^{2}-7 s+4}{s(s-1)^{2}}
$$

Y a continuación hallar la transformada inversa de Laplace $\mathcal{L}^{-1}$, lo que requiere de un arduo trabajo algebraico donde está presente la descomposición en fracciones simples $y$ el uso de las tablas y propiedades de la transformada. Todo este trabajo sumamente engorroso se automatiza usando los operadores solver (solve) e invlaplace que están implementados en Mathcad; para ello se utilizo la función que se ilustra en la Fig. 6, la cual devuelve la expresión analítica $\mathrm{y}(\mathrm{t})$ que define la solución del problema de Cauchy que se está solucionando:

$$
y(t):=\operatorname{MiEc}(Y, s, \text { coefi, Cini })=\operatorname{MdEc}(s) \quad \begin{aligned}
& \text { solve }, Y \\
& \text { invlaplace }
\end{aligned} \rightarrow
$$

Fig. 6. Función implementada en Mathcad que devuelve la solución analitica del problema de Cauchy.

En el caso que nos ocupa resulta:

$$
y(t) \rightarrow 4 \cdot e^{2 \cdot t}-5 \cdot e^{t}-\frac{t^{2} \cdot e^{t}}{2}-t \cdot e^{t}+2
$$

Así, por ejemplo para solucionar el problema de Cauchy

$$
\left\{\begin{array}{l}
y^{\prime \prime}+5 y^{\prime}+6 y=10(1-t) e^{-2 t}+\operatorname{sen}(2 t) \\
y(0)=1, \quad y^{\prime}(0)=2
\end{array}\right.
$$

Tenemos que definir el vector de los coeficientes $\mathrm{C}=$ $(6,5,1)^{\mathrm{T}}$ y el de las condiciones iníciales $\mathrm{CI}=(1,2)^{\mathrm{T}}$; así como la función $f(t)=10(1-t) e^{-2 t}+\operatorname{sen}(2 t)$ correspondiente al segundo miembro de la ecuación diferencial. A partir de aquí el trabajo se reduce a invocar las funciones:

$$
\operatorname{MdEc}(s):=f(t) \text { laplace } \rightarrow \frac{2 \cdot\left(5 \cdot s^{3}+6 \cdot s^{2}+24 \cdot s+24\right)}{(s+2)^{2} \cdot\left(s^{2}+4\right)}
$$

$y(t):=\operatorname{MiEc}(Y, s, C, C l)=\operatorname{MdEc}(s) \quad \begin{aligned} & \text { solve }, Y \\ & \text { invlaplace }\end{aligned} \rightarrow$ 
Por lo que se obtiene la solución del problema de Cauchy, la que viene dada por

$$
\begin{aligned}
y(t) \rightarrow & \frac{206 \cdot e^{-3 \cdot t}}{13}-\frac{59 \cdot e^{-2 \cdot t}}{4}-\frac{5 \cdot \cos (2 \cdot t)}{52}+ \\
& \frac{\sin (2 \cdot t)}{52}+20 \cdot t \cdot e^{-2 \cdot t}-5 \cdot t^{2} \cdot e^{-2 \cdot t}
\end{aligned}
$$

Solución que se ha representado en la Fig. 7

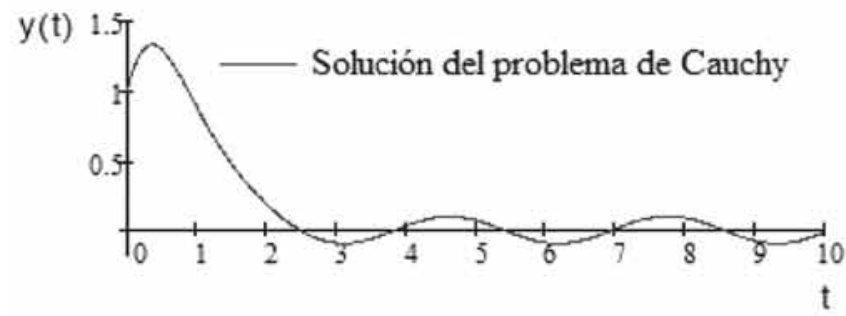

Fig.7. Representación de la solución del problema de Cauchy en el intervalo $[0,10]$

Invitamos al lector que obtenga la solución de este problema de Cauchy usando el proceder clásico; es decir, resolviendo primeramente la ecuación homogénea $y^{\prime \prime}+5 y^{\prime}+6 y=0$ para encontrar $y_{c}(t)$, seguidamente proponiendo y encontrando una solución particular $\mathrm{y}_{\mathrm{p}}(\mathrm{t})$ para $f(t)=10(1-t) e^{-2 t}+\operatorname{sen}(2 t)$ según el método de los coeficientes indeterminados para poder escribir la solución general $\mathrm{y}_{\mathrm{g}}(\mathrm{t})=\mathrm{y}_{\mathrm{c}}(\mathrm{t})+\mathrm{y}_{\mathrm{p}}(\mathrm{t})$; y finalmente aplicar las condiciones iníciales del problema y determinar la solución particular del mismo.

\section{SOLUCIÓN DE PROBLEMAS DE APLICACIONES DE LAS ECUACIONES DIFERENCIALES LINEALES DE SEGUNDO ORDEN}

Como es ampliamente conocido la ecuación diferencial lineal de segundo orden con coeficientes constantes:

$$
a \frac{d^{2} y}{d t^{2}}+b \frac{d y}{d t}+c y=F(t)
$$

Modela problemas oscilatorios, tanto de sistemas vibratorios mecánicos como de circuitos eléctricos RCL (resistencia, condensador e inductancia). En el primer caso y representa la posición del cuerpo en el instante $t$, a corresponde a la masa del cuerpo, $b$ es la constante de amortiguamiento pues dicha fuerza es proporcional a la velocidad, c se identifica con la constante elástica del muelle y $F(t)$ es la fuerza externa que se le aplica al sistema.

En el caso de los circuitos RCL suele tomarse Q para la carga en el instante, variable que ocupa el lugar de y en le ecuación 4, a representa el valor de la inductancia $\mathrm{L}, \mathrm{b}$ es sustituido por la resistencia $\mathrm{R}$, mientras $\mathrm{c}$ es el recíproco de la capacidad $\mathrm{C}$ del condensador y $F(t)$ denota la fuerza electromotriz, que suele denotarse con $E(t)$.

Está claro que si se resuelve un problema de aplicación siguiendo el procedimiento clásico, anteriormente descrito, en una clase pueden ser resueltos un número reducido de problemas; mientras que si se sigue el procedimiento discutido en este artículo se puede resolver una gran variedad de problemas y centrar el trabajo en la modelación del problema y la interpretación de los resultados que son dos momentos muy importantes en el tratamiento de los problemas.

Seguidamente se presentan ejemplos ilustrativos

\section{Ejemplo 1}

Un resorte [11] sujeto a un soporte tiene suspendida una masa de $2 \mathrm{~kg}$ y la constante de elasticidad del resorte es de $4 \mathrm{~N} / \mathrm{m}$. El sistema está en reposo cuando el soporte empieza a oscilar de acuerdo a la expresión $h(t)=$ $2 \cos (3 t)$.

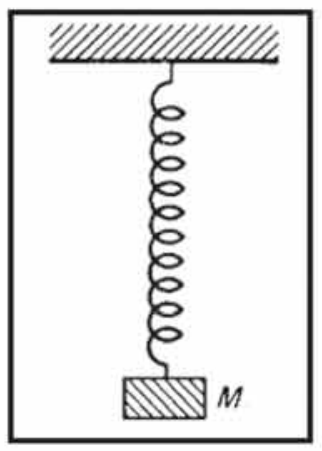

Determine:

a) La ecuación diferencial del movimiento si el sistema completo está inmerso en un medio que opone una fuerza de resistencia numéricamente igual a 6 veces la velocidad instantánea.

b) La ecuación del movimiento (tome en cuenta que el peso está en reposo en la posición de equilibrio cuando el soporte empieza a oscilar).

Rev. Ingeniería, M atemáticas y Ciencias de la Información Vol. 6 / N úm. 12 / julio - diciembre de 2019; pág. 25-35 
c) La gráfica de la ecuación del movimiento.

De la discusión anterior sobre esta tipología de problemas se desprende que el mismo se modela por

$$
\left\{\begin{array}{c}
2 \frac{d^{2} y}{d t^{2}}+6 \frac{d y}{d t}+4 y=2 \cos 3 t \\
y=0, \frac{d y}{d t}=0 \text { para } t=0
\end{array}\right.
$$

La complejidad viene dada cuando el problema de Cauchy es resuelto siguiendo la vía clásica, pero empleando nuestra propuesta, solo hay que definir los vectores coefi $=(4,6,2)^{\mathrm{T}}$ y Ciní $=(0,0)^{\mathrm{T}}$; así como la función $h(t)=2 \cos (3 t)$ y aplicar el procedimiento implementado en Mathcad, obteniendo el resultado que se muestra en la Fig.8, el que conduce al gráfico de la Fig. 9.

$$
\begin{aligned}
\operatorname{MdEc}(s):=h(t) \text { laplace } \rightarrow & \frac{2 \cdot s}{s^{2}+9} \\
y(t):=\operatorname{MiEc}(Y, s, C, C l)=\operatorname{MdEc}(s) & \begin{array}{l}
\text { solve }, Y \\
\text { invlaplace }
\end{array} \rightarrow \\
& \frac{2 \cdot e^{-2 \cdot t}}{13}-\frac{e^{-t}}{10}-\frac{7 \cdot \cos (3 \cdot t)}{130}+\frac{9 \cdot \sin (3 \cdot t)}{130} \\
y(t) \rightarrow & \frac{2 \cdot e^{-2 \cdot t}}{13}-\frac{e^{-t}}{10}-\frac{7 \cdot \cos (3 \cdot t)}{130}+\frac{9 \cdot \sin (3 \cdot t)}{130}
\end{aligned}
$$

Fig. 8. Solución del problema obtenida usando el procedimiento implementado en Mathcad.

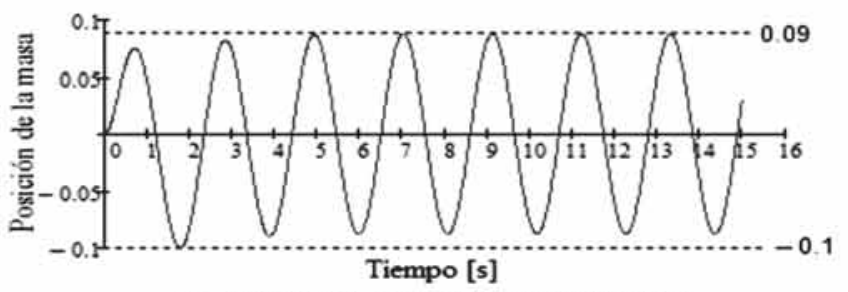

Fig. 9. Gráfica de la ecuación del movimiento.

Como se señaló con anterioridad el procedimiento implementado permite evaluar rápidamente un problema, si por ejemplo se quiere estudiar la influencia de la fuerza amortiguadora, se tiene que cambiar en el vector que contiene los coeficientes de la ecuación diferencial, la componente correspondiente a la fuerza de resistencia en las figuras 10 y 11 se presentan, respectivamente, los resultados obtenidos cuando se considera que la fuerza de resistencia es numéricamente igual a la mitad de la velocidad instantánea y cuando no existe fuerza de resistencia.

$$
\begin{gathered}
y(t) \rightarrow \frac{12 \cdot \sin (3 \cdot t)}{793}-\frac{112 \cdot \cos (3 \cdot t)}{793}+\frac{112 \cdot e^{-\frac{t}{8}} \cdot \cos \left(\frac{\sqrt{127} \cdot t}{8}\right)}{793}- \\
\frac{176 \cdot \sqrt{127} \cdot e^{-\frac{t}{8}} \cdot \sin \left(\frac{\sqrt{127} \cdot t}{8}\right)}{100711}
\end{gathered}
$$

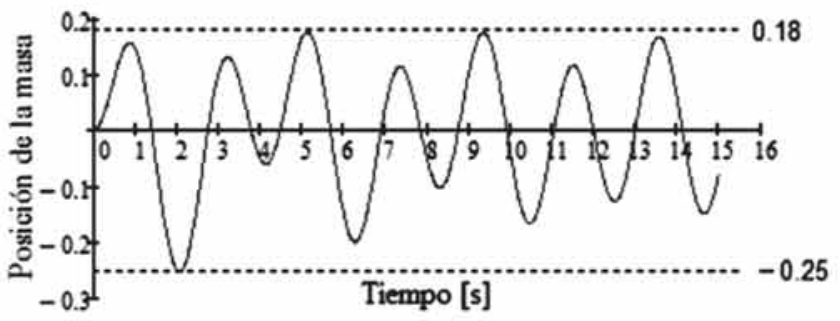

Fig. 10. Ecuación del movimiento y su gráfica cuando la fuerza de resistencia es numéricamente igual a la mitad de la velocidad instantánea, solución obtenida para los coeficientes

$$
\begin{gathered}
C=\left(4, \frac{1}{2}, 2\right)^{T} \\
y(t)=\frac{\cos (\sqrt{2} \cdot t)}{7}-\frac{\cos (3 \cdot t)}{7}
\end{gathered}
$$

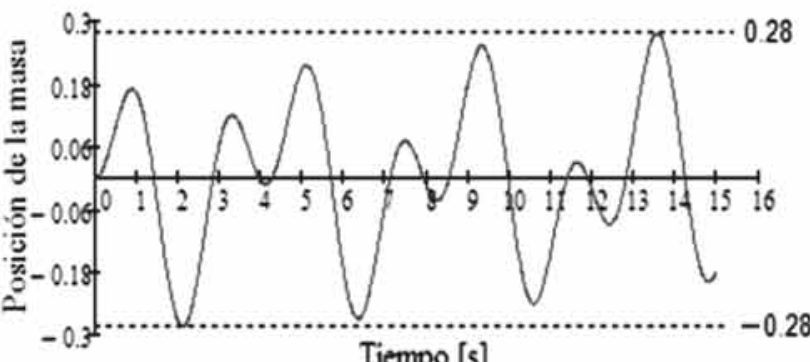

Fig. 11. Ecuación del movimiento y su gráfica cuando la fuerza de resistencia es nula, solución obtenida para los coeficientes de la ecuación diferencial $\mathrm{C}=(4,0,2)$

\section{Ejemplo 2}

Se conectan en serie [12] [13] [14] un inductor de 1 henrio, una resistencia de 2 ohmios, un capacitor de 0.5 faradio y una fuente de voltaje

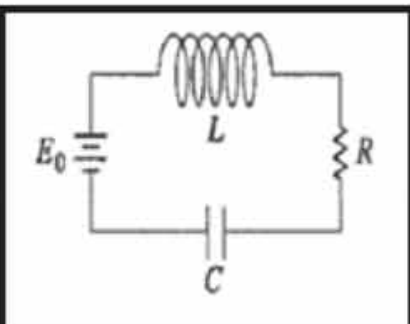


alterno dado por $E(t)=20 \cos (2 t)$ voltios. Si la carga inicial almacenada en el capacitor es de 1 Coulomb y la corriente inicial es igual a cero ampere, encuentre:

a) La carga que contiene el capacitor en el tiempo $t>0$

b) La corriente de estado estacionario

Según lo señalado con anterioridad, este tipo de problema se modela por

$$
\left\{\begin{array}{l}
L \frac{d^{2} Q}{d t^{2}}+R \frac{d Q}{d t}+\frac{Q}{C}=E(t) \\
Q(0)=1, I=\frac{d Q}{d t}=0 \text { para } t=0
\end{array}\right.
$$

En el caso que nos ocupa $L=1, R=2, C=0.5, E(t)=$ $20 \cos (2 t), Q(0)=1$ e $I(0)=0$. Por lo que para el uso de nuestra propuesta hay que definir los vectores Coefi $=\left(\begin{array}{c}1 \\ 0.5\end{array}, 2,1\right)^{T}=(2,2,1)^{T}$ con los coeficientes de la ecuación diferencial y $\operatorname{Cini}=(1,0)^{T}$ correspondiente a las condiciones iníciales. Los resultados del cálculo usando el procedimiento implementado en Mathcad se presentan en la Fig. 12.

$$
\begin{aligned}
& \operatorname{MdEc}(s):=E(t) \text { laplace } \rightarrow \frac{20 \cdot s}{s^{2}+4} \\
& Q(t):=\operatorname{MiEc}(Y, s, \text { Coef, Cini })=\operatorname{MdEc}(s) \quad \begin{array}{l}
\text { solve, }, \\
\text { invlaplace }
\end{array} \\
& Q(t) \rightarrow 4 \cdot \sin (2 \cdot t)-2 \cdot \cos (2 \cdot t)+3 \cdot e^{-t} \cdot \cos (t)-5 \cdot e^{-t} \cdot \sin (t)
\end{aligned}
$$

Fig.12. Resultados de la evaluación del circuito RCL

En el procedimiento analítico del problema se aprecia claramente la solución transitoria y la solución estacionaria y teniendo en cuenta que $I=\frac{d Q}{d t} \quad$ resulta que $I(t)=8 \cos (2 t)+4 \sin (2 t)+2 e^{-t}(\sin t-4 \cos t)$ y la corriente de estado estacionario está dado por $I_{\text {est }}(t)=8 \cos (2 t)+4 \sin (2 t)$. En la Fig. 13 se representan, la carga que contiene el capacitor, la corriente y la corriente de estado estacionario; observe como para valores grandes de $t$, estas dos últimas coinciden.

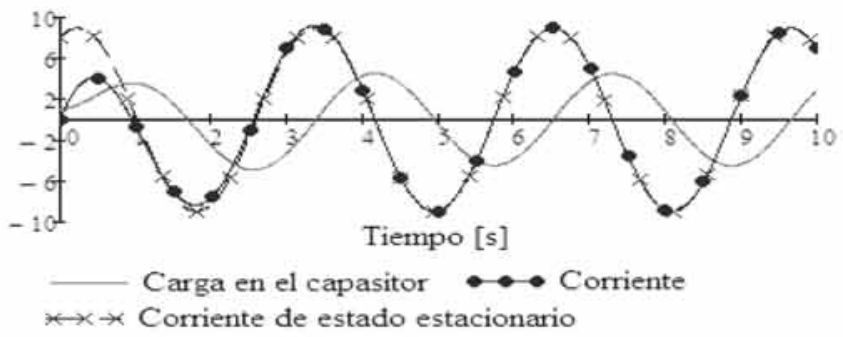

Fig.13. Representación de la carga que contiene el capacitor, la corriente y la corriente de estado estacionario en el circuito RCL.

Como se ilustró en el caso de los problemas de oscilaciones mecánicas, se puede realizar la solución de otras situaciones variando alguno de los parámetros que intervienen en el modelo y si esto se fuera a realizar obteniendo la solución por vía clásica de la ecuación diferencial el proceso consumiría un tiempo considerable. Si por ejemplo queremos analizar la influencia de la fuente de voltaje al sustituir la existente por una de 20 voltios de corriente directa, entonces solo tenemos que sustituir en el cálculo la función $\mathrm{E}(\mathrm{t})=20$. El resultado del cálculo se muestra en la Fig. 14.

$$
\begin{aligned}
& \operatorname{MdEc}(s):=E(t) \text { laplace } \rightarrow \frac{20}{s} \\
& Q(t):=\operatorname{MiEc}(Y, s, \text { Coef }, \text { Cini })=\operatorname{MdEc}(s) \quad \begin{array}{l}
\text { solve }, Y \\
\text { invlaplace }
\end{array} \rightarrow \\
& Q(t) \rightarrow 10-9 \cdot e^{-t} \cdot \sin (t)-9 \cdot e^{-t} \cdot \cos (t) \\
& I(t):=\frac{d}{d t} Q(t) \rightarrow 18 \cdot e^{-t} \cdot \sin (t)
\end{aligned}
$$

Fig. 14. Resultados de la evaluación del circuito RCL considerando una fuente de corriente directa de 20 voltios.

Indudablemente la carga estacionaria en el capacitor es de 10 Coulomb y la corriente de estado estacionario es nula, lo que se observa en la Fig. 15.

Cuando el capacitor se encuentra totalmente cargado, deja de circular corriente por el circuito.

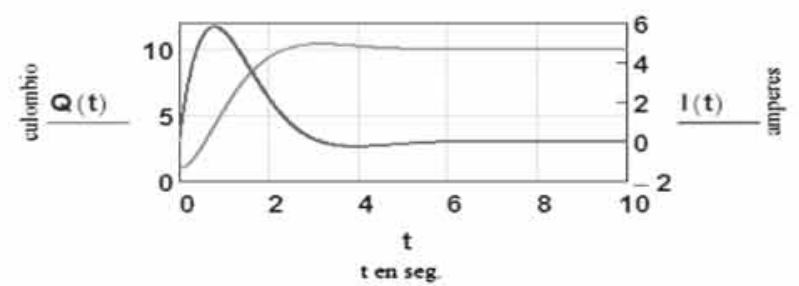

Fig. 15. Representación de la carga que contiene el capacitor y la corriente en el circuito RCL con fuente de corriente directa de 20 voltios. 


\section{Conclusiones}

A partir de las potencialidades para el cálculo simbólico del Mathcad seelaboró un procedimiento basado en el trabajo con la transformada y transformada inversa de Laplace, el cual puede ser utilizado en la solución de problemas con condiciones iníciales para ecuaciones diferenciales lineales con coeficientes constantes. Dicho procedimiento puede ser de gran utilidad en la solución de problemas de aplicación de dichas ecuaciones, pues permite dedicar un mayor tiempo a la modelación del problema y a la discusión de los resultados, automatizando la solución del problema con condiciones iníciales, lo que suel eser extremadamente trabajoso y consume un tiempo apreciable que imposibilita resolver una gran variedad de situaciones en un corto tiempo. Esto fue ilustrado claramente en el trabajo.

\section{RefERENCIAS}

[1] S. Ríos, Modelización. Alianza Universidad, Madrid, 1995.

[2] Luis Zaldívar Henríquez, Yusleydis Cruz López y Michel EnriqueGamboa Graus, “M ediación didáctica contextualizada para la fijación de conceptos matemáticos", Didasc@ia: Didáctica y Educación. Vol. VI. Número 1, Enero-Marzo, ISSN 2224-2643, pp. 49. 2015.
[3] Santiago Vicentey Josetxu Orrantia, "Resolución de problemas y comprensión situacional", Cultura y Educación, 19 (1), ISSN: 1135-6405, pp. 61. 2007.

[4] Pedro Campillo y A ntonio Devesa, "Una experiencia en el uso de un asistente matemático", Revista EMA 2000;5(2):170-181.

[5] Froilan Cajori, A History of Mathematics, The MacMillan Company, London, 1909.

[6] V. Ruel Churchill, Operational Mathematics. McGraw-Hill, London, 1958.

[7] R. Ribnikov, Historia de la Matematica, Editorial MIR, Moscú, 1987

[8] Murray S. Spiegel, Transformadas de Laplace, McGraw-Hill, México, 1967.

[9] Frank Aires, Ecuaciones Diferenciales, McGrawHill, México, 1968.

[10] MathCad Guide. Mathematical Tools N umerical Methods.

[11] David Halliday, Robert Resnick, FISICA, Reverté, Barcelona, 1966.

[12] Francis Weston Sears, Electricity and Magnetism, Addison Wesl ey Company, N ew York, 1962.

[13] Reginald Stevens Kimball, Practical Mathematics, National Educational Alliance, N ew York, 1948.

[14] Edward M. Purcell, Electricity and Magnetism, Berkeley physics course, volume2, Reverté, BarceIona, 1966. 


\section{ANEXO}

Un inductor de 2 henrys, una resistencia de 16 ohmios y un condensador de 0,02 faradios se conectan en serie con una f.e.m. de $E$ voltios. En $t=0$ tanto la carga del condensador como la corriente del circuito valen cero. Encontrar la carga y la corriente en cualquier tiempo

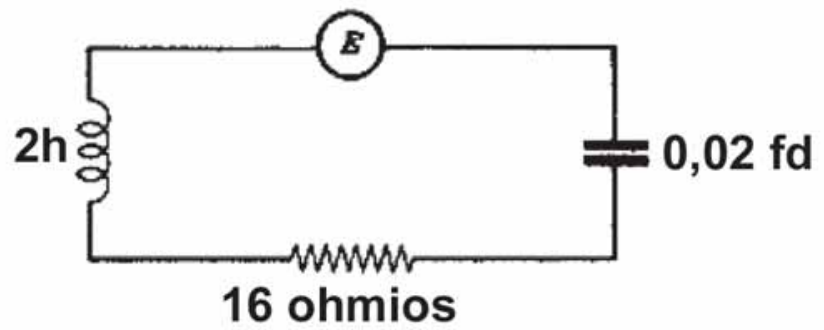
$t>0$ si $E=100$ sen $3 t$ (voltios)

\section{Ecuación diferencial}

$$
2 \frac{d^{2} Q}{d t^{2}}+16 \frac{d Q}{d t}+50 Q=100 \operatorname{sen}(3 t)
$$

$$
2 \frac{d^{2} Q}{d t^{2}}+16 \frac{d Q}{d t}+\frac{1}{0.02} Q=100 \operatorname{sen}(3 t)
$$

\section{Condiciones iniciales}

$$
\begin{aligned}
& Q(0)=0 \\
& Q^{\prime}(0)=0
\end{aligned}
$$

\section{DATOS}

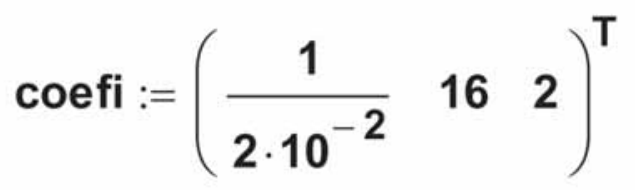

$$
\begin{aligned}
& \text { Cini }:=\left(\begin{array}{ll}
0 & 0
\end{array}\right)^{\top} \\
& f(t):=100 \cdot \sin (3 \cdot t) \\
& k:=2
\end{aligned}
$$




\section{CALCULOS}

$$
\operatorname{Tder}(\mathbf{Y}, \mathbf{s}, \mathbf{k}, \text { Cini }):=\mid \begin{aligned}
& \mathbf{R} \leftarrow \mathbf{Y} \text { if } \mathbf{k}=\mathbf{0} \\
& \mathbf{R} \leftarrow \mathbf{s}^{\mathbf{k}} \cdot \mathbf{Y}-\sum_{\mathbf{i}=0}^{\mathbf{k}-\mathbf{1}}\left(\text { Cini }_{\mathbf{i}} \cdot \mathbf{s}^{\mathbf{k}-\mathbf{i}-1}\right) \text { otherwise }
\end{aligned}
$$

$$
\operatorname{MiEc}(\mathbf{Y}, \mathbf{s}, \text { coefi, Cini }):=\mid \begin{aligned}
& \mathbf{n} \leftarrow \text { last }(\text { coefi }) \\
& \mathbf{R} \leftarrow 0 \\
& \text { for } \mathbf{i} \in \mathbf{0} . . \mathrm{n} \\
& \mathbf{R} \leftarrow \mathbf{R}+\operatorname{coefi} \mathbf{i} \cdot \operatorname{Tder}(\mathbf{Y}, \mathbf{s}, \mathbf{i}, \text { Cini }) \\
& \mathbf{R}
\end{aligned}
$$

$$
\operatorname{MiEc}(\mathbf{Y}, \mathbf{s}, \text { coefi, Cini }):=\mid \begin{aligned}
& \mathbf{n} \leftarrow \text { last }(\text { coefi }) \\
& \mathbf{R} \leftarrow 0 \\
& \text { for } \mathbf{i} \in \mathbf{0} . . \mathrm{n} \\
& \mathbf{R} \leftarrow \mathbf{R}+\operatorname{coefi} \cdot \operatorname{Tder}(\mathbf{Y}, \mathbf{s}, \mathbf{i}, \text { Cini }) \\
& \mathbf{R}
\end{aligned}
$$

$$
\begin{aligned}
& \operatorname{MdEc}(s):=f(t) \text { laplace } \rightarrow \frac{300}{s^{2}+9} \\
& Q(t):=\operatorname{MiEc}(Y, s, \text { coefi }, \operatorname{Cini})=\operatorname{MdEc}(s)
\end{aligned}
$$

$\begin{aligned} & \text { resolver } \\ & \text { invlaplace }\end{aligned} \frac{25 \cdot \sin (3 \cdot t)}{26}-\frac{75 \cdot \cos (3 \cdot t)}{52}+\frac{75 \cdot \cos (3 \cdot t) \cdot e^{-4 \cdot t}}{52}+\frac{25 \cdot \sin (3 \cdot t) \cdot e^{-4 \cdot t}}{26}$

$$
I(t):=\frac{d}{d t} Q(t) \rightarrow \frac{75 \cdot \cos (3 \cdot t)}{26}+\frac{225 \cdot \sin (3 \cdot t)}{52}-\frac{75 \cdot \cos (3 \cdot t) \cdot e^{-4 \cdot t}}{26}-\frac{425 \cdot \sin (3 \cdot t) \cdot e^{-4 \cdot t}}{52}
$$

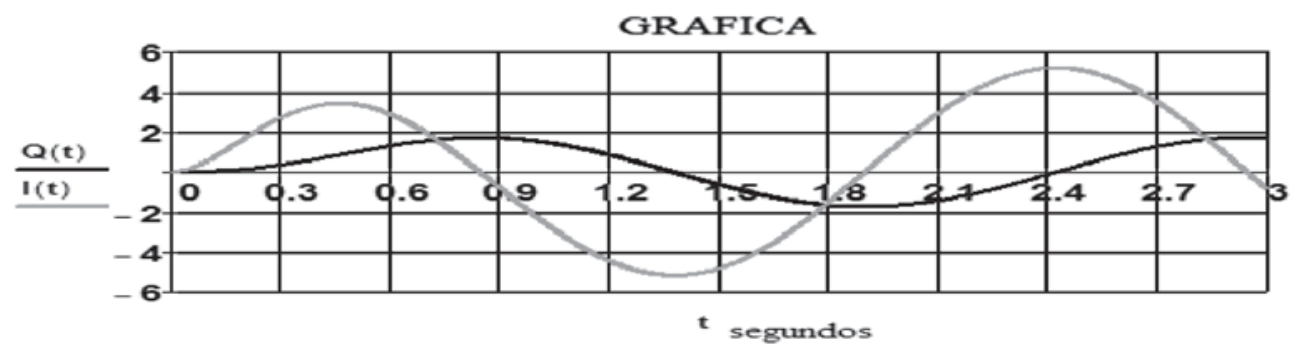


